Atıf için / For Citation: S. Akça, "YBO 3 fosforunun termolüminesans dozimetrik pikinin kinetik parametreleri”, Süleyman Demirel Üniversitesi Fen Edebiyat Fakültesi Fen Dergisi, 15(1), 100-109, 2020.

\title{
YBO3 Fosforunun Termolüminesans Dozimetrik Pikinin Kinetik Parametreleri
}

\author{
Sibel AKÇA ${ }^{* 1}$ \\ ${ }^{I}$ Çukurova Üniversitesi, Fen-Edebiyat Fakültesi, Fizik Bölümü, 01330, Adana, Türkiye \\ *yazışllan yazar e-posta: sakca@cu.edu.tr
}

(Alınış / Received: 17.03.2020, Kabul / Accepted: 15.04.2020, Yaylmlanma / Published: 31.05.2020)

Özet: $\mathrm{Bu}$ çalışmanın amacı, katkısız itriyum borat $\left(\mathrm{YBO}_{3}\right)$ fosforunun termolüminesans (TL) kinetik parametrelerini belirlemektir. Yanma reaksiyonu ile sentezlenen $\mathrm{YBO}_{3}$ fosforu pelet formuna getirilerek TL ölçümleri gerçekleștirilmiștir. Örnekler, 0,1-5 Gy aralığında beta dozuna maruz bırakılarak $2^{\circ} \mathrm{C} / \mathrm{s}^{\prime}$ lik sabit hızla oda sıcaklığından $500^{\circ} \mathrm{C}$ 'ye kadar 1 sıtılmıştır. Örneğe ait TL 1şıma eğrileri, hem farklı dozlar hem de farklı 1sıtma hızları için elde edilmiştir. Elde edilen TL sinyalleri analiz edilerek $210^{\circ} \mathrm{C}$ 'de merkezlenen TL dozimetrik pikinin tuzak parametrelerinin hesaplanması için mevcut pik şekli (PS), farklı ısıtma hızları (VHR) ve ışıma eğrisi ayrıştırma (GCD) metotları kullanılmıştır. Farklı dozlarda sergilenen TL pikine Chen'in PS metodu uygulandığında ortalama aktivasyon enerjisi $(E) \quad 0,847 \mathrm{eV}$, frekans faktörü $(s) 10^{7} \mathrm{~s}^{-1}$ mertebesinde bulunmuştur. İki farklı VHR metodu kullanılarak elde edilen $E$ ve $s$ değerleri, biri için sırasıyla $1,050 \pm 0,05 \mathrm{eV}$ ve $10^{9} \pm 10^{3} \mathrm{~s}^{-1}$ mertebesinde diğeri için $0,890 \mathrm{eV}$ ve $10^{8} \mathrm{~s}^{-1}$ mertebesinde bulunmuştur. GCD metodu R-studio programının "tgcd", paketi kullanılarak uygulanmıştır. Ayrıştırma, 0,5 ve 5 Gy beta dozlarından elde edilen pik maksimuma uygulanmış ve 1 . dereceden kinetiğe uyan iki pikten oluştuğu görülmüştür. Ayrıştırılmış piklerin (pik 1) $E$ değerleri surasıyla 0,889 ve $0,868 \mathrm{eV}$ olarak bulunurken $s$ değerleri ise her ikisi için de $10^{8} \mathrm{~s}^{-1}$ mertebelerinde bulunmuştur.

Anahtar kelimeler: İtriyum borat, Termolüminesans, Aktivasyon enerjisi, Frekans faktörü

\section{Kinetic Parameters of Thermoluminescence Dosimetric Peak of $\mathrm{YBO}_{3}$ Phosphor}

\begin{abstract}
The aim of this study is to determine the thermoluminescence (TL) kinetic parameters of pure yttrium borate $\left(\mathrm{YBO}_{3}\right)$ phosphor. The $\mathrm{YBO}_{3}$ phosphor synthesized by combustion reaction was converted into pellet form and TL measurements were made. The samples exposed to the beta dose in the range of $0.1-5$ Gy were heated from room temperature to $500^{\circ} \mathrm{C}$ at a constant rate of $2^{\circ} \mathrm{C} / \mathrm{s}$. TL glow curves of the sample were obtained for both different doses and various heating rates. The current peak shape (PS), various heating rates (VHR) and glow curve deconvolution (GCD) methods were used to calculate the trap parameters of the TL dosimetric peak centered at $210^{\circ} \mathrm{C}$ by analyzing the obtained TL signals. When Chen's PS method was applied to TL peak displayed in different doses, the average activation energy $(E)$ and frequency factor $(s)$ were found to be $0.847 \mathrm{eV}$ and in the order of $10^{7} \mathrm{~s}^{-1}$. The $E$ and $s$ values obtained using two different VHR methods were $1.050 \pm 0.05 \mathrm{eV}$ and in the order of $10^{9} \pm 10^{3} \mathrm{~s}^{-1}$ for one and $0.890 \mathrm{eV}$ and in the order of $10^{8} \mathrm{~s}^{-1}$ for the other. The GCD method was applied using the 'tgcd' package of the Rstudio program. As a result of applying deconvolution to the peak maximum obtained in beta doses of 0.5 and $5 \mathrm{~Gy}$, two peaks matching the $1^{\text {st }}$ order kinetics were obtained. The $E$ values of the deconvolved peaks (peak 1) were found as 0.889 and $0.868 \mathrm{eV}$ whereas the $s$ values were in the order of $10^{8} \mathrm{~s}^{-1}$.
\end{abstract}

Key words: Yttrium borate, Thermoluminescence, Activation energy, Frequency factor 


\section{Giriș}

Toprak alkali metaller ( $\mathrm{Mg}, \mathrm{Ca}, \mathrm{Ba}$, vb.), geçiş metalleri ( $\mathrm{Zn}, \mathrm{Zr}, \mathrm{Cd}$, vb.) ve nadir toprak elementleri (lantanitler, Sc ve Y) ile oluşturulan borat bileşikleri, doza karşı gösterdikleri doğrusal cevap, tekrar kullanılabilirlik, termolüminesans (TL) sinyalinin uzun ömrü vb. gibi özellikleri nedeniyle dozimetrik malzeme olmaya potansiyel adaylardır. $\mathrm{BaB}_{4} \mathrm{O}_{7}$ [1], $\mathrm{ZnB}_{2} \mathrm{O}_{4}$ [2], $\mathrm{CdB}_{4} \mathrm{O}_{7}$ [3], $\mathrm{MgB}_{4} \mathrm{O}_{7}$ [4] gibi borat bileşiklerinin TL özelliklerinin belirlenmesi amacıyla pek çok çalışma gerçekleştirilmiştir. $\mathrm{Bu}$ borat bileşiklerine özellikle lantanit grubu $\left(\mathrm{Eu}^{+2,+3}, \mathrm{Gd}^{+3}, \mathrm{Nd}^{+3}, \mathrm{~Tb}^{+3}, \mathrm{Sm}^{+3}, \mathrm{Tm}^{+3} \mathrm{vb}\right.$.) aktivatör iyonlar katkılanarak dozimetrik özelliklerinin geliştirilmesi amaçlanmaktadır.

Nadir toprak elementi olan itriyum (Y) ile oluşturulan $\mathrm{Eu}^{+3}, \mathrm{~Tb}^{+3}$ ve $\mathrm{Nd}^{+3}$ katkılı $\mathrm{YBO}_{3}$ bileşikleri üzerine son yıllarda pek çok çalışma yapılmıştır [5-7]. Bu çalışmalar bileşiğin yüksek termal ve kimyasal kararlılığı nedeniyle daha çok fotolüminesans özelliklerini içermektedir. Literatürde $\mathrm{YBO}_{3}$ fosforu ile ilgili sadece birkaç TL çalışması yapılmış olup bunlar da $\mathrm{Eu}^{+3}$ katkılı $\mathrm{YBO}_{3}$ bileşiğinin çeşitli ışınlama türlerinde sergilenen genel TL davranış1 üzerinedir [8-9]. Malzemelerin TL özelliklerinin belirlenmesinde, belirli bir sıcaklıktaki TL sinyalinin kararlılığının bilgisini veren aktivasyon enerjisi ve frekans faktörü gibi temel kinetik parametre hesaplamalarına da başvurulur. Yani, TL olgusunu daha iyi anlamak için TL ölçümlerindeki temel tuzak parametrelerinin belirlenmesi önem taşımaktadır. Bu parametrelerin değerleri, çalışılan malzemenin bazı özel durumlarından kaynaklı olarak anormal değerler de gösterebilir [10]. Kinetik parametrelerin değerlendirilmesi için uygulanan pek çok yöntem vardır. Bunların en bilinenleri, başlangıçtaki artış (IR) [11], pik şekli (PS) [12], farklı 1sıtma hızları (VHR) [13-16] ve 1şıma eğrisi ayrıştırma (GCD) [17] yöntemleridir.

Literatür araştırması yapıldığında saf $\mathrm{YBO}_{3}$ fosforunun genel TL özellikleri ve kinetik parametrelerinin değerlendirilmesi üzerine herhangi bir çalışmanın yapılmadığ görülmektedir. Mevcut çalışmada, yanma yöntemi ile hazırlanan saf $\mathrm{YBO}_{3}$ örneklerinin aktivasyon enerjisi ve frekans faktörü gibi temel tuzak parametreleri PS, iki farklı VHR ve GCD yöntemleri kullanılarak belirlenmiştir.

\section{Materyal ve Metot}

\section{1 $\mathrm{YBO}_{3}$ Fosforunun Yanma Metodu ile Sentezi}

İtriyum Borat $\left(\mathrm{YBO}_{3}\right)$ fosforu yanma yöntemi ile sentezlenmiştir [18-19]. Bu yöntemde

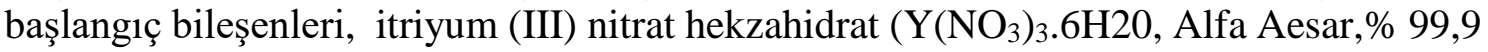
saf), borik asit $\left(\mathrm{H}_{3} \mathrm{BO}_{3}\right.$, Sigma Aldrich, $\geq \%$ 99,5 saf), amonyum nitrat $\left(\mathrm{NH}_{4} \mathrm{NO}_{3}\right.$, Merck, \% 99,9 saf) ve üre $\left(\mathrm{CO}\left(\mathrm{NH}_{2}\right)_{2}\right.$, Merck, \% 99,5 saflık)'dir. Burada amonyum nitrat oksitleyici, üre ise yakıt olarak kullanılmıştır. Toz formundaki bileşenlerin her birinden 0,01 mol alınarak ögütülmek üzere agat havanlara konulmuştur. Öğütme işleminden sonra, bir porselen krozeye konulan karışım, $80^{\circ} \mathrm{C}^{\prime}$ de manyetik bir karıştırıcı üzerinde 20 dakika karıştırılmıştır. $\mathrm{YBO}_{3}$ fosforunu elde etmek üzere karışım $500^{\circ} \mathrm{C}$ 'de 30 dakika firında bekletilerek yanma reaksiyonu gerçekleştirilmiştir.

Yanma reaksiyonu sırasında oluşan gaz halindeki $\mathrm{H}_{2} \mathrm{O}, \mathrm{CO}_{2}$ ve $\mathrm{NO}_{\mathrm{x}}$ buharlaşarak ortamdan uzaklaştırılmıştır. Yanmayı takiben, elde edilen tozlar $900^{\circ} \mathrm{C}$ 'de 1 saat boyunca sinterlenmiş ve normal atmosferik basınç altında aniden oda sıcaklığına soğutulmuştur. Sinterlenmiş toz halindeki fosfor tekrar ögütülerek $90 \mu \mathrm{m}$ 'lik eleklerle elenerek daha küçük parçacıklar haline getirilmiştir. 


\subsection{Deneysel süreç ve Yöntemler}

TL ölçümleri, 0,1 Gys ${ }^{-1}$ 'lık doz hızına sahip ${ }^{90} \mathrm{Sr} /{ }^{90} \mathrm{Y}$ beta 1şınlama, 1sıtma ve PMT (fotoçoğaltıcı tüp) algılama birimlerini içeren Lexsyg Smart TL/OSL okuyucu sistemi ile gerçekleştirilmiştir. Toz $\mathrm{YBO}_{3}$ örnekleri $20 \mathrm{mg}$ tartılarak 2 ton/ $\mathrm{cm}^{2}$ lik basınç altında 10 dakika boyunca $0,70 \mathrm{~mm}$ yüksekliğe ve $6,00 \mathrm{~mm}$ çapa sahip pelet formuna dönüştürülerek TL ölçümleri yapılmıştır. Üç ayrı pelet halinde hazırlanan numunelerin TL sinyalleri, azot ortamında IRSL TL-410 nm'lik optik filtre kullanılarak $2^{\circ} \mathrm{C} / \mathrm{s}^{\prime}$ lik sabit ısıtma hızı ile oda sicaklığından $500^{\circ} \mathrm{C}^{\prime}$ ye kadar ısıtılarak kaydedilmiştir.

Deneysel sürecin ardından, $\mathrm{YBO}_{3}$ örneklerine ait kaydedilen TL sinyalleri analiz edilerek tuzak parametrelerinin belirlenmesi için çeşitli yöntemlere başvurulmuştur. $\mathrm{Bu}$ yöntemlerden en bilinenleri; pik şekli (PS), farklı ısıtma hızları (VHR), başlangıçtaki artış (IR) ve ışıma eğrisi ayrıştırma (GCD) yöntemleridir. Bu yöntemler, TL 1şıma piklerinin doz, 1sıtma hızı, pik maksimum sıcaklığı vb. değişkenlere göre davranışı incelenerek uygulanır. Mevcut çalışmada, $\mathrm{YBO}_{3}$ fosforunun TL kinetik parametrelerinin elde edilmesi için PS, VHR ve GCD metotları kullanılmıştır.

\section{Bulgular}

Hazırlanan $\mathrm{YBO}_{3}$ örneklerinin TL davranışı, 0,1-5 Gy aralığındaki farklı beta ışınlamalarının ardından $2^{\circ} \mathrm{C} / \mathrm{s}^{\prime}$ lik sabit 1 sıtma hızı ile oda sıcaklığından $500^{\circ} \mathrm{C}$ 'ye kadar 1sıtılarak incelenmiştir. Örneklerin TL 1şıma eğrisi, yaklaşık olarak 210 ve $402^{\circ} \mathrm{C}^{\prime} \mathrm{de}$ merkezlenen iki TL pik maksimumdan oluşmaktadır. $210^{\circ} \mathrm{C}$ 'deki keskin TL pikinin dozimetrik pik olduğu ve oldukça yüksek şiddete sahip olduğu görülmüştür (Şekil 1). Şekil 1'de dozimetrik pikin farklı beta dozlarına karşı TL cevabı incelenmiştir. Doza karş1 verilen cevap, $y=b x+a$ denklemine göre $\log I=b \log D+a$ nın uyarlanmas1 şeklinde olup $b=0,95$ olarak bulunmuştur. Burada $I$ TL şiddetini, $D$ dozu, $b$ doğrunun eğimini, $-a$ ise kesişim değerini ifade etmektedir. Elde edilen $0,95^{\prime}$ lik eğim, $\mathrm{YBO}_{3}$ fosforunun doza karşı TL cevabının doğrusal olduğunu yani doza bağımlılığının yüksek olduğunu göstermektedir.

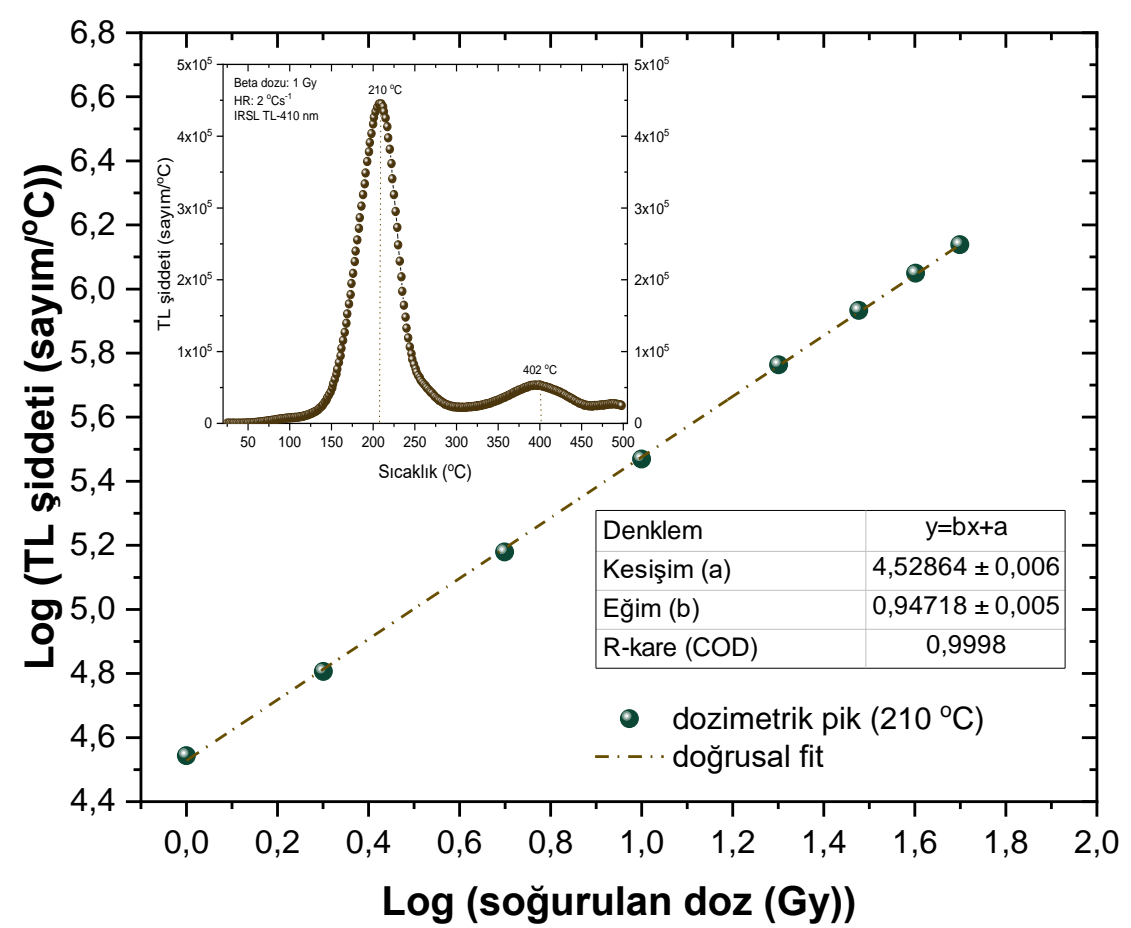

Şekil 1. $\mathrm{YBO}_{3}$ örneğinin TL dozimetrik pikinin farklı beta dozlarına karşı TL cevabı. İçteki şekil, 1 Gy dozda kaydedilen TL 1şıma eğrisini göstermektedir. 
Sentezlenen $\mathrm{YBO}_{3}$ fosforunun $210^{\circ} \mathrm{C}^{\prime}$ de konumlanan TL dozimetrik pikinin aktivasyon enerjisi ve frekans faktörü gibi tuzak parametrelerinin belirlenmesi için PS, VHR ve GCD yöntemleri uygulanmıştır. Bu yöntemler ve sonuçları aşağıda detaylı olarak verilmiştir.

\subsection{PS Yöntemi}

TL 1şıma pikinden yararlanılarak temel parametrelerin değerlendirilmesine olanak sağlayan en temel yöntem, pik şekli (PS) yöntemi olarak bilinir. PS yöntemi, doğrudan TL pikinin şekline dayanır. Yöntemde bilinmesi gerekenler Şekil 2'de detaylandırılmıştır. Şekil 2'de görülen $\tau=T_{m}-T_{1}$ pikin düşük sıcaklık kısmına ait yarı genişliği, $\delta=T_{2}-T_{m}$ ise yüksek sıcaklık kısmına ait yarı genişliği ifade etmektedir. Bu yöntemde, $\mu=\delta / \omega$ simetri faktörü 0,42 ise TL piki 1 . dereceden kinetiğe, 0,52 ise 2 . dereceden kinetiğe sahiptir. Bu çalışmada Chen'e ait PS yöntemi uygulanmış olup, bu yöntem $0,1 \mathrm{eV}$ ve $2,0 \mathrm{eV}$ arasında geniş bir enerji yelpazesi ve $10^{5}$ ile $10^{13} \mathrm{~s}^{-1}$ arasındaki frekans faktörleri için uygundur [20].

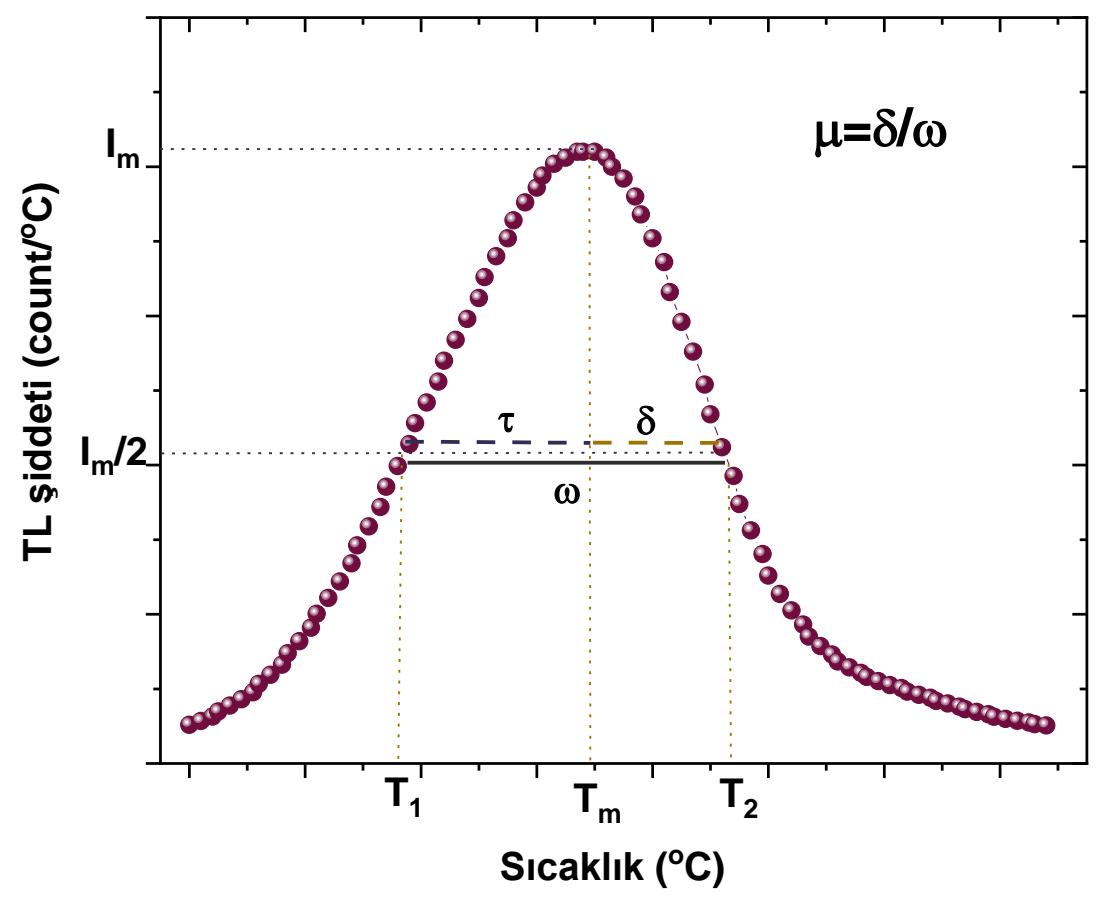

Şekil 2. PS yöntemini karakterize eden parametreler

$\mathrm{YBO}_{3}$ fosforunun $0,1-5 \mathrm{~Gy}$ doz aralığında $2^{\circ} \mathrm{C} / \mathrm{s}^{\prime}$ lik sabit 1 sitma hızı ile 1sitılarak $210^{\circ} \mathrm{C}^{\prime}$ de gözlenen TL dozimetrik pik maksimum incelenerek simetri faktörü $\mu$ ve Chen'in PS denklemi kullanılarak $E$ ve $s$ değerleri hesaplanmıştır [12,21]. 0,1-5 Gy arasındaki her bir beta dozu ışınlamasında, dozimetrik pik maksimumun $\mu$ ve $E$ değerleri Şekil 3'deki gibi bir değişim göstermiştir. Şekil 3'den görüldügü gibi $\mu$ değeri 0,42 ve 0,44 aralığında değișmekte olup bu değerler dozimetrik pik maksimumun 1. dereceden kinetiğe sahip olduğuna işaret etmektedir. Chen'in PS denkleminden elde edilen aktivasyon enerjisi varyasyonları olan $E_{\tau}, E_{\omega}$ ve $E_{\delta}$ 'nın ortalaması olan $E$ değerleri 0,83$0,90 \mathrm{eV}$ aralığında bulunmuştur. 


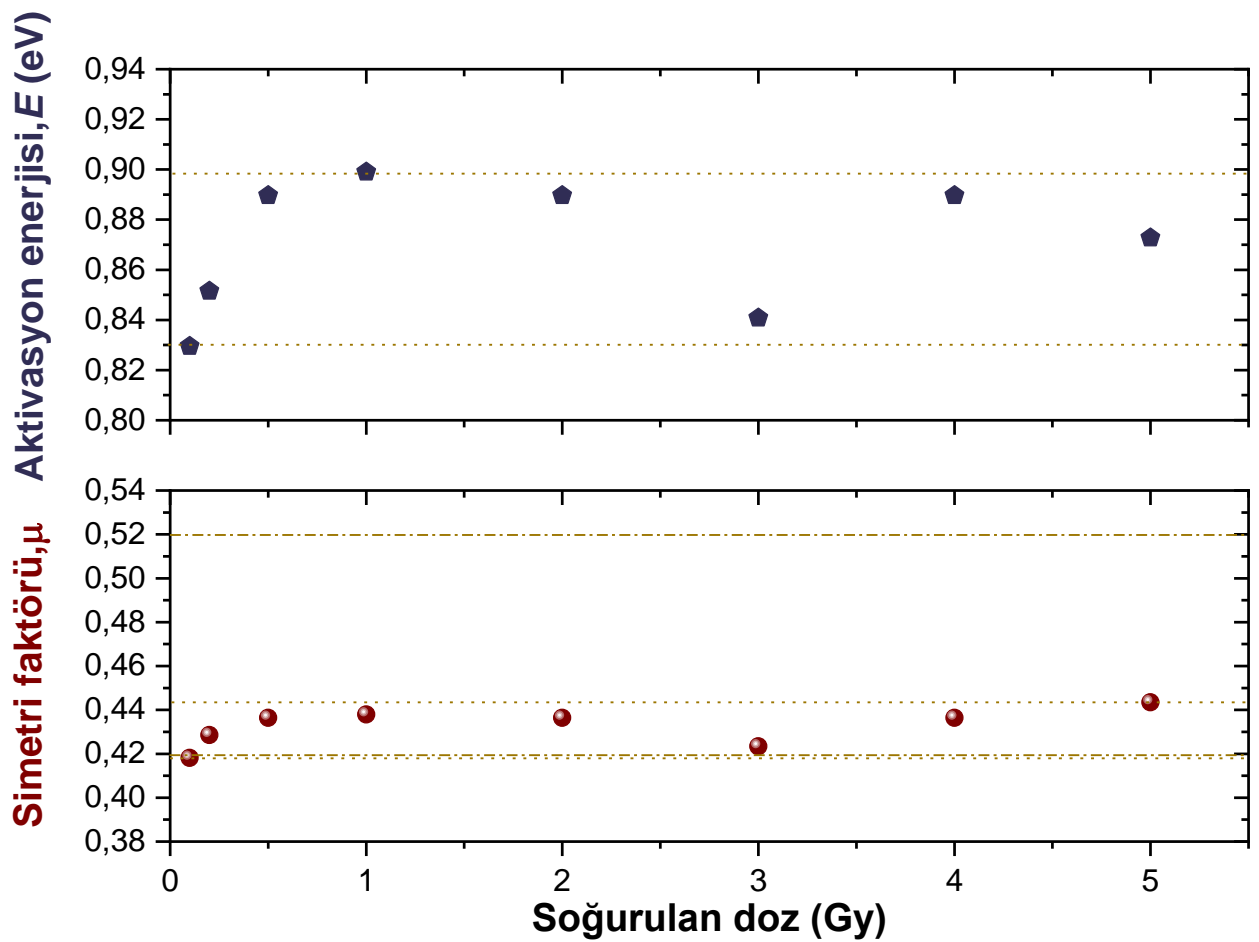

Şekil 3. 0,1-5 Gy aralığındaki dozlara karşı dozimetrik pik maksimumun $\mu$ ve $E$ değerlerinin değişimi

Dozimetrik pik maksimumun farklı doz miktarları için, Chen düzeltmeli PS Metodu kullanılarak elde edilen kinetik parametrelerinin ortalaması ise Tablo 1'de verilmiştir. Bu sonuçlara göre ortalama $E$ ve $s$ değerleri sırasılyla $0,847 \mathrm{eV}$ ve $6,21 \times 10^{7} \mathrm{~s}^{-1}$ olarak bulunmuştur.

Tablo 1. 0,1-5 Gy arasında değişen dozlarda dozimetrik pik maksimuma ait Chen düzeltmeli PS metodu kullanılarak elde edilen kinetik parametrelerin ortalaması

\begin{tabular}{cccccccccc}
\hline $\boldsymbol{T}_{\boldsymbol{m}}\left({ }^{\mathbf{0}} \mathbf{C}\right)$ & $\boldsymbol{\tau}$ & $\boldsymbol{\omega}$ & $\boldsymbol{\delta}$ & $\boldsymbol{\mu}=\boldsymbol{\delta} / \boldsymbol{\omega}$ & $\boldsymbol{E}_{\boldsymbol{\tau}}$ & $\boldsymbol{E}_{\boldsymbol{\omega}}$ & $\boldsymbol{E}_{\boldsymbol{\delta}}$ & $\boldsymbol{E}(\boldsymbol{e} \boldsymbol{V})$ & $s\left(\boldsymbol{s}^{-1}\right)$ \\
\hline 210 & 32,0 & 55,6 & 23,7 & $\mathbf{0 , 4 2 5}$ & 0,829 & 0,849 & 0,865 & $\mathbf{0 , 8 4 7}$ & $\mathbf{6 , 2 1 \times 1 0 ^ { 7 }}$ \\
\hline
\end{tabular}

\subsection{VHR Yöntemi}

\subsubsection{Hoogenstraaten VHR Yöntemi}

Hoogenstraaten tarafindan önerilen [13] farklı 1sıtma hızları (VHR) yöntemi, $\mathrm{YBO}_{3}$ fosforunun $E$ ve $s$ gibi kinetik parametrelerini tahmin etmek için uygulandı. Bu yöntem, 1sıtma hızı (HR) arttırıldığında pik maksimum sıcaklığının $\left(T_{m}\right)$ yüksek sıcaklıklara doğru kaymasından faydalanılarak uygulanır. Yöntem, birinci dereceden kinetiğe sahip TL 1şıma eğrileri için önerilmiş olmasına rağmen, bugüne kadar yapılan çalışmalar diğer kinetik mertebedeki piklere de uygulanabileceğini göstermiştir [20,22]. Bu yöntemde kinetik parametreler, $\ln \left(T_{m}^{2} / \beta\right)$ ve $\left(1 / T_{m}\right)$ arasındaki doğrusal ilişkiden yararlanılarak elde edilir. Hoogenstraaten'ın VHR yöntemi ile $E$ ve $s$ değerleri aşağıda verilen Denklem 1 kullanılarak hesaplanır.

$$
\ln \left(\frac{T_{m}^{2}}{\beta}\right)=\left(\frac{E}{k}\right)\left(\frac{1}{T_{m}}\right)+\ln \left(\frac{E}{k s}\right)
$$

Denklem 1'de $T_{m}(\mathrm{~K})$, her $\beta(\mathrm{K} / \mathrm{s})$ 1sıtma hızındaki pik maksimum sıcaklığını, $s\left(\mathrm{~s}^{-1}\right)$ frekans faktörünü, $E(\mathrm{eV})$ aktivasyon enerjisini ifade eder. $k$ boltzman sabiti olup değeri $8.617 \times 10^{-5}(\mathrm{eV} / \mathrm{K})$ 'dir. Bu nedenle, Denklem 1'deki sicaklık değerleri Kelvin birimine 
çevrilmiştir. Bu denklem, $\ln \left(T_{m}^{2} / \beta\right)$ 'nin $\left(1 / T_{m}\right)$ 'ye karşı grafiği çizildiğinde elde edilen doğrunun eğiminin $\left(\frac{E}{k}\right)$ 'ya kesişimin $\ln \left(\frac{E}{k s}\right)$ 'ye eşit olduğunu ifade eder. Böylelikle, $E$ ve $s$ değerleri hesaplanabilir.

Bu çalışmada, VHR yöntemi ile kinetik parametrelerin hesaplanabilmesi için 0,5 ve $7^{\circ} \mathrm{C} / \mathrm{s}$ arasında değişen sekiz farklı 1sıtma hızının $\mathrm{YBO}_{3}$ fosforunun TL 1şıma eğrisi üzerine etkisi incelenmiştir. Isıtma hızının, TL sinyalleri üzerine etkisi incelenirken sıcaklık gecikmesi (TLA) etkisi de göz önünde bulundurulmaya çalışılmıştır. TLA etkisi, TL ölçümü esnasında ısıl çift ile örnek arasındaki sıcaklık farkından kaynaklanmakta olup, bu etki ile $T_{m}$ değeri daha yüksek sicaklıklara kaymaktadır. Bu nedenle, Kitis ve Tuyn tarafından [23] önerilen sicaklık düzeltmesi formülü kullanılarak $T_{m}$ değerlerinde düzeltme yapılmıştır. $\mathrm{YBO}_{3}$ fosforunun dozimetrik pikinin $0,5 \mathrm{ve} 7^{\circ} \mathrm{C} / \mathrm{s}$ aralığındaki farklı 1sıtma hızlarında $\ln \left(T_{m}^{2} / \beta\right)$ 'nin $\left(1 / T_{m}\right)$ 'ye karşı grafiği Şekil 4'de verilmiştir. Hem ölçülen hem de düzeltilen $T_{m}$ değerleri için hesaplama yapılmıştır. Şekil 4'den de görüldüğü gibi ölçülen ve hesaplanan değerler için lineer fitlerin korelasyonu $R^{2}$ sirasıyla 0,98 ve 0,99 olup iyi bir korelasyon içindedir.

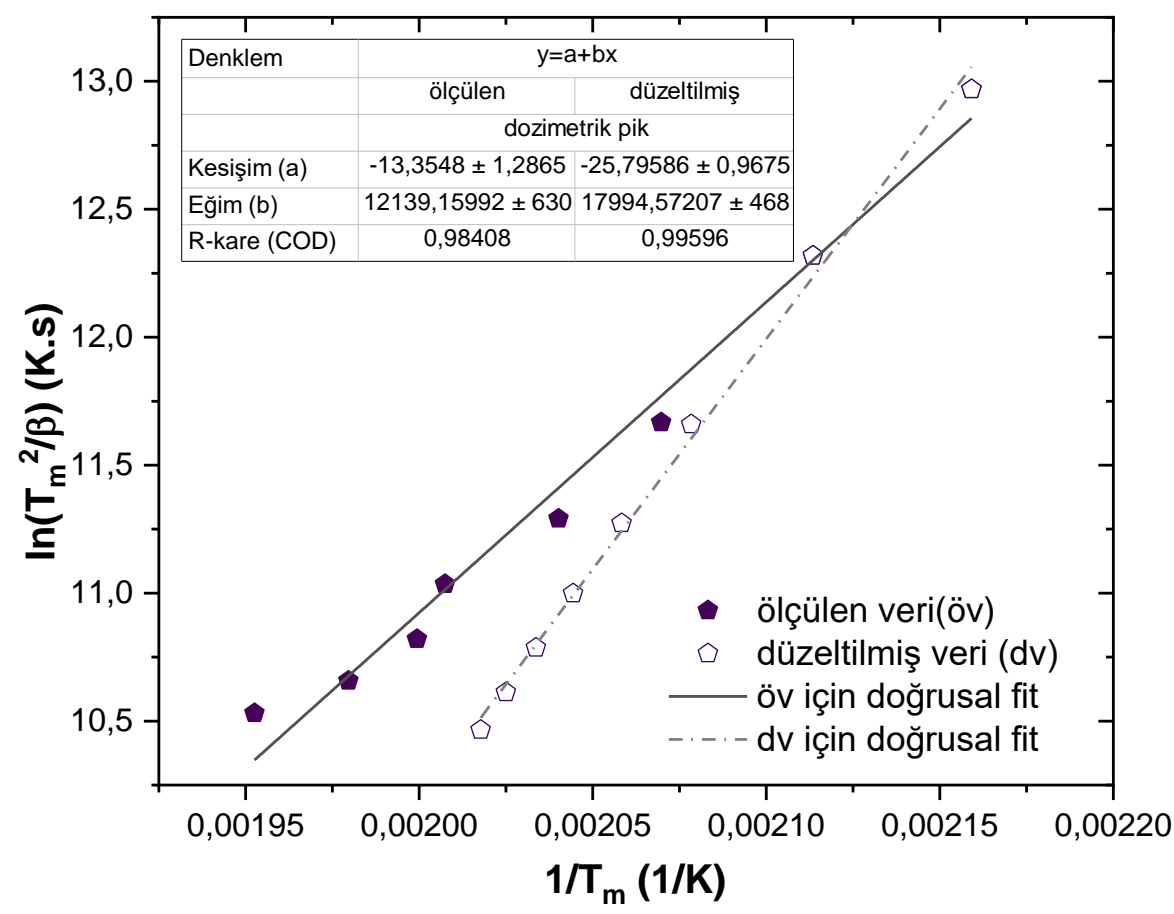

Şekil 4. Dozimetrik pik maksimumun $0,5-7^{\circ} \mathrm{C} / \mathrm{s}$ aralığındaki farklı ısıtma hızları için $\ln \left(T_{m}^{2} / \beta\right)$ 'nin $\left(1 / T_{m}\right)$ 'ye karşı değişimi

Şekil 4'deki doğruların eğim ve kesişim değerleri, Denklem 1'de yerine konularak Tablo 2 'de verilen $E$ ve $s$ değerleri belirlenmiştir. Elde edilen $E$ değeri ölçülen $T_{m}$ değeri için $1,05 \pm 0,05 \mathrm{eV}$, düzeltilmiş $T_{m}$ değeri için 1,55 $\pm 0,04 \mathrm{eV}$ olarak bulunmuştur. Elde edilen $s$ değerleri ise ölçülen ve düzeltilen $T_{m}$ değerleri için sırasıyla $10^{9} \pm 10^{3}$ ve $10^{15} \pm 10^{3}$ mertebelerinde bulunmuştur.

Tablo 2. Hoogenstraaten'e ait VHR metodu kullanılarak elde edilen ölçülmüş ve düzeltilmiş $E$ ve $s$ değerleri

\begin{tabular}{cccc}
\hline \multicolumn{2}{c}{ Ölçülen değerler } & \multicolumn{2}{c}{ Düzeltilmiş değerler } \\
$\boldsymbol{E}(\boldsymbol{e} \boldsymbol{V})$ & $\boldsymbol{s}\left(\boldsymbol{s}^{-1}\right)$ & $\boldsymbol{E}(\boldsymbol{e} \boldsymbol{V})$ & $\boldsymbol{s}\left(\boldsymbol{s}^{-1}\right)$ \\
\hline $1,05 \pm 0,05$ & $7,66 \times 10^{9} \pm 2,29 \times 10^{3}$ & $1,55 \pm 0,04$ & $3,87 \times 10^{15} \pm 1,23 \times 10^{3}$ \\
\hline
\end{tabular}




\subsubsection{Booth, Bohum ve Porfianovitch VHR Yöntemi}

Booth (1954) [14], Bohum (1954) [15] ve Porfianovitch (1954) [16] birbirlerinden bağımsız yaptıkları çalışmalarla $E$ ve $s$ kinetik parametrelerini 1. dereceden kinetikler için değerlendirmişlerdir. $E$ değeri, $\beta_{1}$ ve $\beta_{2}$ gibi iki farklı 1sıtma hızlarına karşılık gelen $T_{m 1}$ ve $T_{m 2}$ pik maksimum sıcaklıklarının değişimine dayandırılır [20].

$$
E=\frac{k T_{m 1} T_{m 2}}{T_{m 1}-T_{m 2}} \ln \left(\frac{\beta_{1}}{\beta_{2}}\left(T_{m 2}-T_{m 1}\right)^{2}\right)
$$

$E^{\prime}$ 'nin bilinmesi ile $s$ değeri aşağıdaki denklem yardımı ile bulunur:

$$
s=\frac{E}{k} \exp \left\{\left[T_{m 2} \ln \frac{T_{m 2}^{2}}{\beta_{2}}-\ln \frac{T_{m 1}^{2}}{\beta_{1}}\right] /\left(T_{m 1}-T_{m 2}\right)\right\}
$$

Denklem 2 ve 3 kullanılarak $\mathrm{YBO}_{3}$ fosforunun dozimetrik pikinin $\left(210^{\circ} \mathrm{C}\right) E$ ve $S$ değerleri hesaplanmıştır. Bu değerler aşağıdaki Tablo 3'de verilmiştir.

Tablo 3. Booth, Bohum ve Porfianovitch'e ait VHR metodu kullanılarak elde edilen $E$ ve $s$ değerleri

\begin{tabular}{cccccccc}
\hline $\boldsymbol{\beta}_{\mathbf{1}}$ & $\boldsymbol{\beta}_{\mathbf{2}}$ & $\boldsymbol{E}(\boldsymbol{e} \boldsymbol{V})$ & $\boldsymbol{s}\left(\boldsymbol{s}^{-1}\right)$ & $\boldsymbol{\beta}_{\mathbf{1}}$ & $\boldsymbol{\beta}_{\mathbf{2}}$ & $\boldsymbol{E}(\boldsymbol{e} \boldsymbol{V})$ & $\boldsymbol{s}\left(\boldsymbol{s}^{-\boldsymbol{1}}\right)$ \\
\hline 0,5 & 7 & 1,016 & $3,18 \times 10^{9}$ & 2 & 4 & 0,873 & $1,13 \times 10^{8}$ \\
2 & 6 & 0,964 & $1,11 \times 10^{9}$ & 2 & 7 & 0,835 & $4,27 \times 10^{7}$ \\
3 & 6 & 0,900 & $2,34 \times 10^{8}$ & 3 & 5 & 0,993 & $2,36 \times 10^{9}$ \\
4 & 7 & 0,791 & $1,51 \times 10^{7}$ & 3 & 7 & 0,746 & $5,13 \times 10^{6}$ \\
\hline \multicolumn{4}{c}{} & $\boldsymbol{E}_{\text {ort. }}=\mathbf{0 , 8 9 0} \pm \mathbf{0 , 0 9}$ & & \multicolumn{3}{c}{$\boldsymbol{s}_{\text {ort. }}=\mathbf{8 , 8 2 \times 1 0 ^ { 8 }}$} \\
\hline
\end{tabular}

Tablo 3'den görüldüğ̈̈ gibi, farklı 1sıtma hızlarından elde edilen $E$ değerleri 0.746-0.993 $\mathrm{eV}$ arasında değişmekte olup $0.890 \mathrm{eV}$ 'luk ortalama değere sahiptir. Elde edilen $s$ değerleri ise $10^{6}-10^{9}$ mertebesi arasında değişmekte olup ortalaması $8.82 \times 10^{8} \mathrm{~s}^{-1}$ bulunmuştur.

\subsection{GCD Yöntemi}

$\mathrm{YBO}_{3}$ fosforunun kinetik parametre hesab1, $210^{\circ} \mathrm{C}$ 'de merkezlenen dozimetrik pik maksimumun tek bir ışıma pikinden oluştuğu varsayılarak yapılmıştır. Bu maksimumun tam olarak kaç bileşenin kombinasyonu olduğunu kontrol etmek için Işıma Eğrisi Ayrıştırma (GCD) yöntemi uygulanmıştır. Dozimetrik pik maksimum, R-studio programında bulunan "tgcd", paketi kullanılarak ayrıştırılmıştır [24]. Şekil 5'te sentezlenmiş $\mathrm{YBO}_{3}$ örneğinin 0,5 ve 5 Gy beta dozlarında sergilediği dozimetrik pik maksimumun iki ayrıştırılmış pikten oluştuğu görülmektedir. Ayrıştırılmış bu pikler yaklaşık olarak 207 (pik 1) ve 242 (pik 2) ${ }^{\circ} \mathrm{C}$ 'lerde maksimum vermektedirler. Ayrıştırılmış pikler detaylı incelendiğinde, pik 1'in dozimetrik maksimumun özelliklerini taşıdığ pik 2'nin ise çok anlamlı olmayıp yüksek sıcaklık pikine geçişten dolayı ortaya çıktığı anlaşılmaktadır. Bu ayrıştırma 1. dereceden kinetik teoriye göre yapılmış olup 0,5 ve 5 Gy beta dozlarında deneysel verilerle fit eğrisi arasındaki uyumu ifade eden FOM değerleri sırasıyla 2,05 ve 2,17 olarak elde edilmiştir. Pik 1'i simgeleyen tuzakların aktivasyon enerjisi 0,5 ve 5 Gy'lik dozlar için sırasıyla 0,889 ve $0,868 \mathrm{eV}$ olarak bulunmuştur. İyonlaştırıcı radyasyona maruz bırakıldıktan sonra elektronların tuzaklardan kaçış olasılığını ifade eden frekans faktörü değeri ise 0,5 ve 5 Gy'lik dozlar için sirasılyla $1,95 \times 10^{8}$ ve $1,14 \times 10^{8}$ dir (Pik 1 için). 


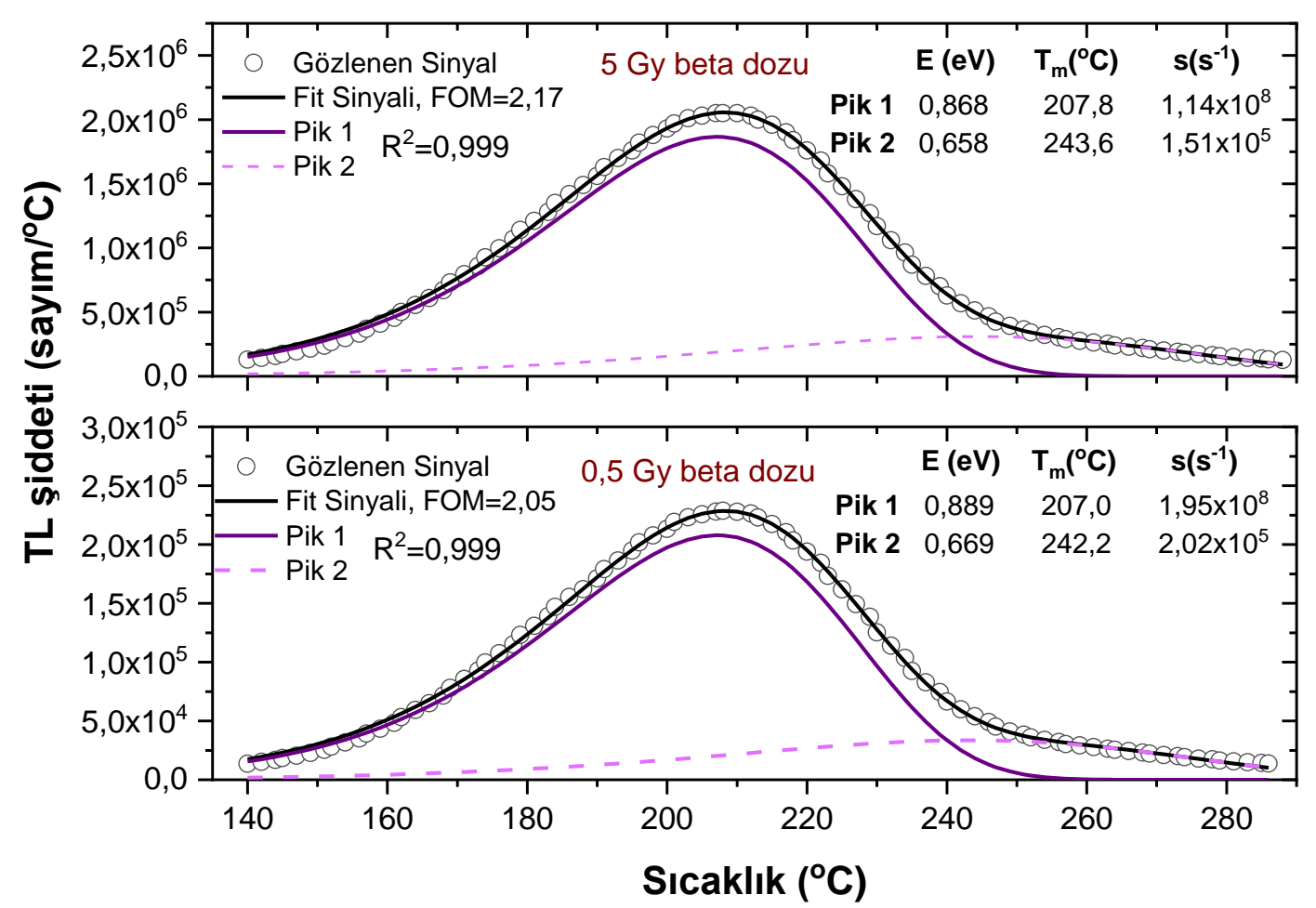

Şekil 5. 0,5 ve 5 Gy beta dozlarında TL dozimetrik pik maksimumun R-studio ile GCD yöntemi kullanılarak elde edilen ayrıştırılmış pikleri ve tuzak parametreleri

Öte yandan, PS metodu, Chen'in denklemi kullanılarak temel ayrıştırılmış pike (pik 1) tekrar uygulanmıştır. Pik 1 'in 0,5 ve 5 Gy dozlarda PS metodu uygulanarak elde edilen kinetik parametreleri Tablo 4'te verilmiştir. Tablo 4'e göre, 0,5 ve 5 Gy dozda pik 1 için $E$ değeri sırasıyla yaklaşık olarak 0,92 ve $0,89 \mathrm{eV}$ olarak hesaplanmıştır. Frekans faktörü $s$ ise her iki doz için de $10^{8}$ mertebesinde bulunmuştur.

$\mathrm{Bu}$ çalışmada kullanılan bütün yöntemler kullanılarak elde edilen frekans faktörü değerleri incelendiğinde $10^{6}-10^{9} \mathrm{~s}^{-1}$ aralığında bulunmuştur. Bu değerlerin, $10^{12}-10^{14} \mathrm{~s}^{-1}$ aralığında olan örgü titreşim frekansı değerinden daha küçük olduğu görülmektedir. Düşük frekans faktörünün nedeninin, elektronların iletim bandına geçiş yapmadan lokal geçişlerle 1şıma gerçekleşmesi şeklinde olduğu düşünülür $[25,26]$.

Tablo 4. R-studio programı ile 0,5 ve 5 Gy’lik dozlarda ayrıştırılmış piklerin (pik 1) Chen düzeltmeli PS Metodu kullanilarak elde edilen kinetik parametreleri

\begin{tabular}{ccccccccccc}
\hline & $\boldsymbol{T}_{\boldsymbol{m}}\left({ }^{\mathbf{0}} \mathbf{C}\right)$ & $\boldsymbol{\tau}$ & $\boldsymbol{\omega}$ & $\boldsymbol{\delta}$ & $\boldsymbol{\mu}=\boldsymbol{\delta} / \boldsymbol{\omega}$ & $\boldsymbol{E}_{\boldsymbol{\tau}}$ & $\boldsymbol{E}_{\boldsymbol{\omega}}$ & $\boldsymbol{E}_{\boldsymbol{\delta}}$ & $\boldsymbol{E}(\boldsymbol{e} \boldsymbol{V})$ & $\boldsymbol{s}\left(\boldsymbol{s}^{-1}\right)$ \\
\hline $\mathbf{0 , 5 G y}$ & 207 & 29,5 & 51,5 & 22,0 & 0,427 & 0,899 & 0,919 & 0,930 & 0,916 & $3,86 \times 10^{8}$ \\
$\mathbf{5 G y}$ & 207 & 30,2 & 52,7 & 22,5 & 0,427 & 0,874 & 0,894 & 0,906 & 0,891 & $2,04 \times 10^{8}$ \\
\hline
\end{tabular}

\section{Sonuç ve Yorum}

Yanma reaksiyonu ile sentezlenen katkısız $\mathrm{YBO}_{3}$ fosforunun termolüminesans (TL) dozimetrik pik maksimumun kinetik parametrelerini belirlemek için 0,1-5 Gy aralığında farklı dozlarda $2^{\circ} \mathrm{C} / \mathrm{s}^{\prime}$ lik sabit hızla ve 0,5 Gy'lik beta 1 şınlamasıyla $0,5-7^{\circ} \mathrm{C} / \mathrm{s}^{\prime}$ lik farklı 1sıtma hızlarında TL 1şıma pikleri kaydedilmiştir. $210^{\circ} \mathrm{C}$ 'de konumlanan TL dozimetrik maksimumun aktivasyon enerjisi $(E)$ ve frekans faktörü $(s)$ gibi parametre tayini pik şekli (PS), farklı 1sıtma hızları (VHR) ve ışıma eğrisi ayrıştırma (GCD) yöntemleri kullanılarak yapılmıştır. 0,1-5 Gy aralığındaki çeşitli dozlarda elde edilen TL pik maksimuma PS metodu uygulanmış ve simetri faktörü $(\mu)$ 0,42-0,44 aralığında bulunarak 1.dereceden kinetiğe sahip olduğu görülmüştür. Chen'in PS denklemi kullanılarak 
ortalama aktivasyon enerjisi $(E)$ ve frekans faktörü $(s)$ değerleri sirasıyla $0,847 \mathrm{eV}$ ve $10^{7} \mathrm{~s}^{-1}$ mertebesinde bulunmuştur. Hoogenstraaten'e ait VHR metodu kullanılarak elde edilen $E$ ve $S$ değerleri sirasiyla $1,050 \pm 0,05 \mathrm{eV}$ ve $10^{9} \pm 10^{3} \mathrm{~s}^{-1}$ mertebesinde, Booth, Bohum ve Porfianovitch'e ait VHR metodu kullanılarak elde edilen değerlerin ortalamas1 ise sırasiyla $0,890 \mathrm{eV}$ ve $10^{8} \mathrm{~s}^{-1}$ mertebesinde bulunmuştur. R-studio programının " tgcd"' paketi kullanılarak uygulanan GCD metodunda, 0,5 ve 5 Gy beta dozlarında elde edilen TL pik maksimum 1. dereceden kinetiğe uyan üst üste binmiş pikten oluştuğu görülmüştür. $\mathrm{Bu}$ ayrıştırılmış pikler (pik 1 ve pik 2) her iki doz için de benzer sicaklıklarda elde edilmiştir. Dozimetrik pik maksimum ile benzer sıcaklığa sahip olan yaklaşık $207^{\circ} \mathrm{C}$ 'deki ayrıştırılmış pikin (pik 1) her iki doz için FOM değeri 2,05 ve 2,17 olarak elde edilmiştir. 0,5 ve 5 Gy'lik dozlar için $E$ değerleri sırasıyla 0,889 ve $0,868 \mathrm{eV}$ olarak bulunurken $s$ değerleri her ikisi için $10^{8} \mathrm{~s}^{-1}$ mertebelerinde bulunmuştur. $\mathrm{Bu}$ sonuçlara göre, yanma yöntemi ile sentezlenen saf $\mathrm{YBO}_{3}$ fosforunun dozimetrik piki 1 . dereceden kinetiğe uymaktadır. Yani, dozimetrik piki temsil eden tuzaklarda bulunan elektronlar serbest kaldığında tekrar tuzaklanmadan ya yeniden birleşme merkezinde bir deşikle birleşir ya da değerlik bandına gider. Booth, Bohum ve Porfianovitch'e ait VHR metodu kullanılarak elde edilen enerji değerlerinin ortalaması, GCD metodu uygulanarak elde edilen sonuçlarla oldukça iyi uyum içindedir. $\mathrm{Bu}$ da, $\mathrm{YBO}_{3}$ örneğinin dozimetrik pikini simgeleyen tuzağın derinliğinin yaklaşık olarak $0,87-0,89 \mathrm{eV}$ olduğunu göstermektedir. Ayrıca, yöntemlerde elde edilen düşük frekans faktörünün, elektronların iletim bandına geçmeden lokal geçişlerle 1şıma yapmasından kaynaklandığ düşünülmektedir.

\section{Araştırmacıların Katkı Oranı Beyanı}

Sibel Akça: Araştırma, Materyal/Malzeme Temini, Orijinal Taslak Yazımı, İnceleme ve Düzenleme

\section{Destek ve Teşekkür Beyanı}

$\mathrm{Bu}$ çalışma süresi boyunca yaptığı her türlü bilimsel katkılarından dolayı Çukurova Üniversitesi Fizik Bölümü öğretim üyesi Prof. Dr. Mustafa Topaksu'ya ve malzeme hazırlama sürecindeki katkılarından dolayı Çukurova Üniversitesi Seramik Bölümü öğretim üyesi Dr. Y. Ziya Halefoğlu'na en içten teşekkürlerimi sunarım.

\section{Çatışma Beyanı}

Bu çalışmanın yazarları olarak herhangi bir çatışma beyanımız bulunmadığını bildiririz.

\section{Etik Kurul Onayı ve/veya Aydınlatılmış Onam Bilgileri}

Bu çalışmanın yazarları olarak herhangi bir etik kurul onayı ve/veya aydınlatılmış onam bilgileri beyanımız bulunmadığını bildiririz.

\section{Kaynakça}

[1] A. N. Yazici, M. Dogan, V. E. Kafadar, and H. Toktamis, "Thermoluminescence of undoped and Cedoped $\mathrm{BaB}_{4} \mathrm{O}_{7}$," Nucl. Instrum. Methods Phys. Res., B 246 (2), 402-408, 2006.

[2] T. Dogan, L. Tormo, S. Akca, N. Kucuk, J. Garcia Guinea, Y. Karabulut, M. Ayvacikli, M. Oglakci, M. Topaksu, and N. Can, "Cathodoluminescence and thermoluminescence of $\mathrm{ZnB}_{2} \mathrm{O}_{4}: \mathrm{Eu}^{3+}$ phosphors prepared via wet-chemical synthesis," Ceram. Int., 45, 4918-4925, 2019.

[3] T. N. Khamaganova, T. G. Khumaeva, A. K. Subanakov, and A. V. Perevalov, "Synthesis and thermoluminescence properties of $\mathrm{CdB}_{4} \mathrm{O}_{7}: \mathrm{Tb}^{3+}$ and $\mathrm{CdB}_{4} \mathrm{O}_{7}: \mathrm{Mn}^{2+}$. Inorg. Mater., 53, 81-85, 2017.

[4] V. Pagonis, N. Brown, G.S. Polymeris, and G. Kitis, "Comprehensive analysis of thermoluminescence signals in MgB4O7:Dy,Na dosimeter,” J. Lumin., 213, 334-342, 2019.

[5] R. Balakrishnaiah, Yi. S. Soo, K. Jang, S. Ho Lee, B.K. Moon, and J.H. Jeong, "Enhanced luminescence properties of $\mathrm{YBO}_{3}: \mathrm{Eu}^{3+}$ phosphors by Li-doping," Mater. Res. Bull. 46, 621-626, 2011.

[6] R. G. Nair, S. Nigam, V. Sudarsan, R. K. Vatsa, and V. K. Jain, "YBO ${ }_{3}$ versus $\mathrm{Y}_{3} \mathrm{BO}_{6}$ host on $\mathrm{Tb}^{3+}$ luminescence," J. Lumin., 195, 271-277, 2018. 
[7] L. J. Q. Maia, A. L. Mourab, J. Vladimir, Cid B. de Araújo, "Structural properties and near infrared photoluminescence of $\mathrm{Nd}^{3+}$ doped $\mathrm{YBO}_{3}$ nanocrystals," Opt. Mater., 95, 109227, 2019.

[8] V. Dubey, J. Kaur, S. Agrawal, N.S. Suryanarayana, K.V.R. Murthy, "Effect of Eu ${ }^{3+}$ concentration on photoluminescence and thermoluminescence behavior of $\mathrm{YBO}_{3}: \mathrm{Eu}^{3+}$ phosphor," Superlattice Microst., 67, 156-171, 2014.

[9] V. Dubey, N. V. Dubey, S. J. Dhoble, and H. C. Swart, "TL glow curve analysis and kinetics of UV, $\beta$ and $\gamma$ irradiated $\mathrm{YBO}_{3}: \mathrm{Eu}^{3+}$ and $\mathrm{Y}_{2} \mathrm{O}_{3}: \mathrm{Eu}^{3+}$ phosphors," J. Mater. Sci: Mater. Electron., 28, 1356513578, 2017.

[10] R. Chen, V. Pagonis, J. L. Lawless, "Evaluated thermoluminescence trapping parameters-What do they really mean?," Radiat. Meas., 91, 21-27, 2016.

[11] G.F.J. Garlick, A.F. Gibson, "The electron trap mechanism of luminescence in sulphide and silicate phosphors," Proc. Phys. Soc., 60 (6), 574-590, 1948.

[12] R. Chen and S.A.A. Winer, "Effects of heating rates on glow curves," J. Appl. Phys., 41, 5222755232, 1970.

[13] W. Hoogenstraaten, "Electron Traps in Zinc Sulphide Phospors," Philips Res. Repts., 13, 515-693, 1958.

[14] A.H. Booth, "Calculation of electron trap depths from thermoluminescence maxima," Canad. J. Chem., 32, 214-215, 1954.

[15] A .Bohun, "Thermoemission und photoemission von Natriumchlorid," Czech. J. Phys. 4, 91-93, 1954.

[16] I. A. Parfianovich, "On the determination of the energy depth of capture levels in crystal phosphors," J. Exp. Theor. Phys., SSSR 26, 696-703, 1954.

[17] R. Chen, S. W .S. McKeever, Theory of Thermoluminescence and Related Phenomena, Singapore: World Scientific, 1997, pp. 272.

[18]S. Akça, Z. G. Portakal, T. Dogan, N. Kucuk, A. Canimoglu, M. Topaksu, N. Can, "Thermoluminescence properties of $\mathrm{Tb}$ doped $\mathrm{Mg}_{2} \mathrm{SiO}_{4}$ after beta irradiation," Nucl. Instrum. Methods Phys. Res., B 458, 12-20, 2019.

[19] Y. Z. Halefoglu, "Luminescent properties and characterisation of $\mathrm{LaB}_{3} \mathrm{O}_{6}: \mathrm{Eu}^{3+}$ phosphor synthesized using the combustion method," Appl. Radiat. Isot., 148, 40-44, 2019.

[20]C. Furetta, Handbook of Thermoluminescence, World Scientific publishing Co. Pre.Ltd., 2003, pp. 260-263, pp. 440, pp.435

[21]R. Chen and Y. Kirsh, Analysis of Thermally Stimulated Processes. New York: Pergamon Publishing Co. Pvt. Ltd., 1981, pp. 162.

[22] S. W. S. McKeever, Thermoluminescence of Solids, Cambridge: Cambridge Univ. Press., 1985, pp. 90-92.

[23] G. Kitis, and J.W.N. Tuyn, "A simple method to correct for the temperature lag in TL glow-curve measurements," J. Phys. D Appl. Phys., 31, 2065-2073, 1998.

[24] J. Peng, Z. Dong and F. Han, "tgcd: An R package for analyzing thermoluminescence glow curves," SoftwareX, 5, 112-120, 2016.

[25] S. W. S. McKeever, M. Moscovitch, and P. D. Townsend, Thermoluminescence Dosimetry Materials: Properties and Uses, Ashford, United Kingdom: Nuclear Technology Publishing, 1995, pp. 63-66.

[26] Y. Jin, Y. Hu, L. Chen, X. Wang, Z. Mou, G. Ju, and F. Liang, "Luminescent properties of a reddish orange emitting long-lasting phosphor CaO:Pr ${ }^{3+}, "$ Mater. Sci. Eng. B, 178(18), 1205-1211, 2013. 